\title{
A Regra dos Sinais para a Multiplicação: ponto de encontro com a noção de congruência semântica e o princípio de extensão em matemática*
}

\section{Sign Rule for Multiplication: the notion of semantic congruence and the extension principle in mathematics}

\author{
Méricles T. Moretti**
}

\section{Resumo}

A regra dos sinais para a multiplicação é apresentada, sem demonstração, por Diofanto de Alexandria há muito tempo. Somente em 1867 ela é demonstrada por Hankel como, sendo a única que possui a vantagem de satisfazer a distributividade à esquerda e a distributividade à direita. Assim, ele resolve o problema definitivamente do ponto de vista matemático quando usa o princípio de extensão ao prolongar para os números negativos a propriedade da distributividade, há muitos anos utilizada para os positivos. Veremos que a questão do ponto de vista didático se mantém ainda hoje, e apontamos, também, estudos na perspectiva de ensino dessa regra baseada na ideia de congruência semântica e no princípio de extensão em matemática.

Palavras-chave: Regra dos Sinais. Congruência Semântica. Diofanto de Alexandria. Teorema de Hankel. Princípio de Extensão.

\footnotetext{
"Apoio CNPq e CAPES.

** Doutor em Didática da Matemática pela Universidade Louis Pasteur, Estrasburgo. Professor do Programa de Pós-Graduação em Educação Científica e Tecnológica e do Departamento de Matemática da Universidade Federal de Santa Catarina, Campus Universitário do Bairro Trindade (PPGECT/ MTM/UFSC), CEP: 88040-900, cidade de Florianópolis em Santa Catarina. Endereço para correspondência: Rua João Mussi, n. 248, Bairro Carianos, CEP: 88047-510, Florianópolis, SC, Brasil.E-mail: mthmoretti@gmail.com.
} 


\begin{abstract}
In the $3^{\text {rd }}$ century B.C., the sign rule for multiplication was presented by Diophantus of Alexandria without any demonstration. Only in 1867 was the rule demonstrated by Hankel as being the only one with the advantage of satisfying both left and right distributivity. Thus, Hankel solves the problem from the mathematical point of view when he uses the extension principle to apply the distributivity property, which has been used for many years with positive numbers, to negative numbers. This paper shows that this approach is still present in mathematics teaching today. The paper also presents studies from a teaching perspective of this rule based on the idea of semantic congruence and the extension principle in mathematics.
\end{abstract}

Keywords: Sign Rule. Semantic Congruence. Diophantus.Hankel’s Theorem. Extension Principle.

\title{
1 Introdução
}

O germe da regra dos sinais para a multiplicação, tal como a conhecemos hoje, é, em geral, atribuído a Diofanto de Alexandria ${ }^{1}$. Este algebrista escreveu um pequeno ensaio sobre números poligonais; um tratado de álgebra que nos chegou em condições bastante precárias; e um trabalho sobre porismas, o qual se perdeu (BALL, 1960, p. 104).

A regra que estabelece que " $-x-=+\mathrm{e}-x+=-$ " aparece em seu tratado de álgebra, de forma explícita em Diofanto de Alexandria (2007a, p. 22): "Menos multiplicado por menos é mais e menos por mais é menos". A regra usual dos sinais para a multiplicação aparece, ainda, de forma transitória na sua obra, em várias relações algébricas, sem que alguma justificativa seja dada. A relação algébrica ${ }^{2}$ seguinte

$$
(a-b)(c-d)=a c-a d-b c+b d
$$

que é atribuída a Diofanto de Alexandria, apresenta todas as possibilidades de sinais para a regra usual da multiplicação (BALL, 1960, p. 106).

\footnotetext{
${ }^{1}$ Alguns autores, entre eles Glaeser (1981, p. 311), consideram que Diofanto de Alexandria viveu no período que vai do ano 250 até 350 depois de Cristo. Há divergências entre os pesquisadores quanto a este período.

${ }^{2}$ Essa relação que apresenta todas as possibilidades de sinais para a regra usual da multiplicação não a encontramos nas referências de Diofanto de Alexandria (2007A e 2007b).
} 
A regra usual dos sinais para a multiplicação, só muito mais tarde, em 1867, foi demonstrada por Hankel como sendo a única das regras possíveis, aquela que preserva as distributividades à esquerda e à direita. Hoje, do ponto de vista estritamente matemático, este resultado não causa nenhuma dificuldade ou estranheza. No entanto, resta ainda a questão didático-pedagógica do seu uso e explicação.

Ao longo da história a regra dos sinais para a multiplicação, em particular, para o caso " $-\times-=+$ " foi a que mais causou perplexidade. Um exemplo desta perplexidade é encontrado em Euler (1770) ${ }^{3}$ citado por GLAESER (1981, p. $319,320)$, que, em uma obra pedagógica com o objetivo de fornecer uma explicação para a regra dos sinais para a multiplicação, utiliza um argumento do tipo lógico: para justificar os casos “ $+x+=+$ ", “ $-x+=-$ " e “ $+x-=-$ " ele usa a ideia de ganho e dívida, respectivamente, para os números positivos e negativos, e, para o caso " $-x-$ " é atribuído o resultado “+” com a justificativa para manter o mesmo número de sinais positivos e negativos nos resultados desses quatro casos de multiplicações.

Glaeser (1981, p. 329) enfatiza, ainda, em vários momentos do seu artigo, a dificuldade ao longo da história de o homem lidar com os números negativos, e cita como primor de exemplo, do que ele chama de "desejo de evitar os negativos", o caso da escala de temperatura de Fahrenheit, construída no século XVIII. Fahrenheit tomou como origem de sua escala, não o ponto de fusão do gelo, como fez Celsius, mas a temperatura mais baixa que conheceu no inverno de 1709, em Danzig. A temperatura aproximada do corpo humano foi o segundo ponto da sua escala, que a dividiu em 100 graus e que resultou nos valores de 32 e 212 graus, respectivamente, para os pontos de fusão e ebulição da água. A temperatura de três dígitos, como era referida na época, $100^{\circ} \mathrm{F}\left(37,78^{\circ} \mathrm{C}\right)$, tornouse ponto de referência para aplicação de medicação nos pacientes. Não imaginava Fahrenheit que, em anos seguintes, a temperatura em um dia do inverno chegaria a um nível mais baixo do que ele havia proposto como limite inferior para o zero na sua escala centígrada (KARLSON, 1961, p. 40, 41).

O "desejo de evitar os negativos" que Glaeser (1981, p.329) refere-se à possibilidade pensada por Fahrenheit de que a temperatura informada fosse sempre acima de zero ou graus de calor como era utilizado, na época, em oposição aos graus de frio para as temperaturas abaixo de zero.

\footnotetext{
${ }^{3}$ EULER, Léonard. Vollständige Anleitung zur Algebra Opera Omnia. Series Prima. Volumen Primum, 1911.
} 


\section{0 princípio da extensão: a regra usual para a multiplicação dos números relativos e os números imaginários}

A explicação do porque da regra dos sinais está no Teorema de Hankel. Dentre as várias possibilidades de regra dos sinais para a multiplicação, a escolha recaiu sobre a regra que preserva as distributividades à esquerda e à direita. Estas propriedades já são observadas quando tratamos com os números positivos, e é bastante natural procurarmos prolongá-las também para o caso dos negativos. Assim, as propriedades da distributividade à direita e distributividade à esquerda, que gozam os números positivos, são estendidas, também, para o caso dos números negativos desde que mantenhamos a regra usual para a multiplicação. Esta ideia de prolongamento baseia-se em um princípio, que em parte orienta o trabalho intelectual do homem, que Caraça $(1951$, p. 9, 10) denomina de princípio de extensão:

[...] o homem tem tendência a generalizar e estender todas as aquisições do seu pensamento, seja qual for o caminho pelo qual essas aquisições se obtêm, e a procurar o maior rendimento possível dessas generalizações pela exploração metódica de todas as suas consequências. (CARAÇA, 1951, p. 10).

Os números positivos gozam da propriedade das distributividades à direita e à esquerda. Por este princípio, para que os números negativos também mantenham estas propriedades, a regra dos sinais deve ser definida do modo como conhecemos atualmente.

O princípio da extensão pode explicar muitas conquistas em matemática. Para o homem antigo, os números naturais estavam ligados aos objetos de que se servia para contar. Já em nossos dias, o número natural pode estar desligado das coisas e se tornar algo puramente aritmético. Uma das consequências do desprendimento dos números naturais das coisas é o surgimento, por exemplo, da noção de infinito, a noção de que não há um número natural maior do que os outros pela "possibilidade de repetição ilimitado do ato mental - juntar uma unidade" (CARAÇA, 1951, p. 11).

A aplicação do princípio da extensão à regra dos sinais usuais, o caso “$\times-=+$ " em particular, usada sem demonstração, por muitos séculos, possibilitou a criação de um novo conjunto dos números imaginários:

Em consequência da regra dos sinais, um número negativo não tem para raiz quadrada nenhum número real. A 
necessidade de levantar esta exceção, de modo a tornar possíveis todas as operações sobre números reais, teve como resultado a criação dos números complexos. Esta nova classe de números é, portanto, de origem algébrica. (COSTA, 1971, p. 222).

A razão é bastante simples conforme apontamos nesta citação: jamais um número real multiplicado por ele mesmo tem como produto um número real negativo, desde que adotemos a regra usual dos sinais para a multiplicação. Caso diferente seria se tivéssemos assumido a regra para o caso " $-\times-=-$ ", poderíamos escrever, por exemplo, que $\sqrt{-4}=-2$ uma vez que, para este caso $(-2) \times(-2)=-4$. O caso das raízes de índices ímpares se manteria inalterado, por exemplo, $\sqrt[3]{-27}=-3$, pois $(-3) \times(-3) \times(-3)=-27$.

Por que os estudiosos da época mantiveram a regra “ $-x-=+$ ” e, com isso, perderam a possibilidade de extrair a raiz de qualquer índice de números negativos ou positivos? Esta atitude permitiu a criação de um novo conjunto de números, os imaginários: a equação seguinte $\mathrm{x}^{3}-15 \mathrm{x}-4=0$ tem $\mathrm{x}=4$ como uma das raízes (se pode verificar por substituição direta na equação). No entanto, a fórmula de Cardano aplicada a esta equação dá a seguinte solução $\mathrm{x}=\sqrt[3]{2+\sqrt{-121}}+\sqrt[3]{2-\sqrt{-121}}$. Como explicar o aparecimento de $\sqrt{-121}$ na solução que tem $x=4$ como uma das soluções? Fato como este deve ter levado os estudiosos da época a não procederem a alteração na regra dos sinais para o caso " $-\times-$ ", mas, em lugar disso, eles procuraram encontrar uma forma de tratar com estes números que, mais tarde, seriam chamados de imaginários ${ }^{3}$.

A regra usual da multiplicação já era usada há muito tempo, desde Diofanto de Alexandria (2007a, 2007b) sem que fosse demonstrada. A superação da raiz de índice par (por exemplo, a raiz quadrada) de número negativo surge quando da resolução de equações, por volta do século XIV. Caso fosse possível admitir a raiz quadrada de um número negativo (no campo dos reais), seria necessário desconstruir muito do que já havia sido elaborado em matemática até aquele momento. Prevaleceu, então, a ideia de estender a propriedade de distributividade dos números positivos para os negativos, no lugar de poder extrair a raiz de qualquer índice de um número negativo. Foi preciso a criação do conjunto

\footnotetext{
${ }^{4}$ Com a engenhosa ideia de $\mathrm{i}=\sqrt{-1}, \mathrm{i}^{2}=-1, \mathrm{i}^{3}=-\mathrm{i}, \mathrm{i}^{4}=1$ e assim por diante, a equação pode ser manipulada e com isso a raiz $\mathrm{x}=4$ obtida: para $\mathrm{o}$ caso da equação dada basta constatar que $\sqrt[3]{2-\sqrt{-121}}+\sqrt[3]{2+\sqrt{-121}}=(2-i)+(2+i)=4$.
} 
dos números complexos para que a raiz de índice par pudesse também ser estendida aos números negativos. De qualquer forma, o princípio da extensão foi aplicado nos dois casos:

- com manutenção da regra usual para a multiplicação e com isso as distributividades à esquerda e à direita puderam ser estendidas aos números negativos;

- com a manutenção da regra usual, a raiz de índice par de um número negativo não pôde ser extraída no campo dos números reais. Isso permitiu a criação do conjunto dos complexos, que tem em sua concepção a ideia da extensão da raiz de índice par para os números negativos. Assim, no universo do conjunto dos números complexos é possível extrair a raiz de qualquer índice de um número positivo, negativo ou mesmo complexo.

\section{Como aparecem os números negativos nos PCN e NCTM}

Nos Parâmetros Curriculares Nacionais de Matemática - PCN para o ensino fundamental das séries iniciais (BRASIL, 1998a) os números negativos são citados em quatro momentos: um deles fala da importância da compreensão da constituição dos números, da existência das diferentes categorias, ao longo da história. A relação entre medida e um número é outro aspecto citado que possibilitaria a compreensão da criação dos diversos tipos de número, entre eles, os negativos.

Nos PCN para o ensino fundamental de $5^{\mathrm{a}}$ a $8^{\mathrm{a}}$ séries (BRASIL, 1998b) os números negativos são citados em quatro páginas, aproximadamente. Tratam, inicialmente, de um pequeno histórico, e apresentam um quadro de obstáculos esperados quando da abordagem desses números. São eles:

- conferir significado às quantidades negativas;

- reconhecer a existência de números nos dois sentidos a partir do zero, enquanto para os naturais a sucessão acontece num único sentido;

- reconhecer diferentes papéis para o zero (zero absoluto e zero-origem);

- perceber a lógica dos números negativos que contraria a lógica dos números naturais - por exemplo, é possível "adicionar 6 a um número e obter 1 no resultado", como também é possível "subtrair um número de 2 e obter 9"; - interpretar sentenças do tipo $\mathrm{x}=-\mathrm{y}$, (o aluno costuma pensar que x é positivo e y é negativo) (BRASIL, 1998b, p. 98). 
Uma vez que os números negativos são interpretados como uma ampliação dos naturais e incorporados às leis da aritmética, nos PCN há a recomendação de usar a representação da reta numérica para os números inteiros. Apresentam, ainda, a regra dos sinais por meio de uma tabela a ser completada, sugerindo regularidades das sequências construídas, observando um padrão numérico (acrescentar 3 ou retirar 3).

Nas Normas (NCTM, 2008, p.172 e 175) para os três primeiros anos de escolaridade uma das expectativas é o desenvolvimento do sentido dos números inteiros, sem referência explícita aos inteiros negativos. Estes só aparecem com clareza nas expectativas para os $3^{\circ}, 4^{\circ}$ e $5^{\circ}$ anos de escolaridade: explorar números inteiros inferiores a zero com o prolongamento da reta numérica e de aplicações familiares. Por aplicações familiares são citados exemplos de temperatura e dívidas em dinheiro. Novamente, nos três anos seguintes, $6^{\circ}, 7^{\circ}$ e $8^{\circ}$ anos de escolaridade, há referência à compreensão dos inteiros relativos sem, no entanto, especificar algo relacionado aos negativos.

Nesses documentos, os PCN para o ensino fundamental e NCTM, há diversas indicações para a compreensão dos números negativos que se restringem, mais especificamente, à sua compreensão para o campo aditivo. A regra dos sinais para a multiplicação é somente sugerida nos PCN para as últimas séries do ensino fundamental.

\section{Alguns modelos didáticos de explicação da regra dos sinais}

A seguir, apresentamos os principais modelos inspirados no desenvolvimento histórico dos números relativos para explicar a regra dos sinais para a multiplicação: geométricos baseados na relação de Diofanto de Alexandria, modelos baseados no prolongamento dos naturais e modelos baseados na ideia de balanço de dupla, por exemplo, do tipo ganho/perda, muitas vezes denominado de modelo comercial.

Para aliviar as notações ou para deixar mais transparente o procedimento aplicado, poderemos utilizar ou não os parênteses nas operações com os inteiros.

\subsection{Modelos de área baseados na relação de Diofanto de Alexandria}

Este modelo justifica a regra dos sinais para a multiplicação, utilizando a relação atribuída a Diofanto de Alexandria: $(\mathrm{a}-\mathrm{b})(\mathrm{c}-\mathrm{d})=\mathrm{ac}-\mathrm{ad}-\mathrm{bc}+\mathrm{bd}$.

Em Hogben (1956, p. 287 - 288) encontramos a figura seguinte para 
chegar à relação de Diofanto de Alexandria:

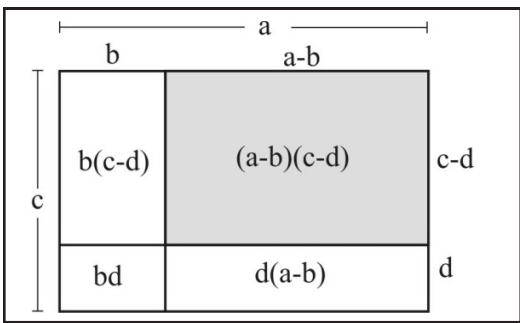

Figura 1 - modelo geométrico para se chegar à relação de Diofanto de Alexandria

Na Figura 1, em termos de área, podemos escrever:

$$
a c=(a-b)(c-d)+b(c-d)+d(a-b)+b d
$$

$\mathrm{ou}$

$$
(a-b)(c-d)=a c-a d-b c+b d
$$

que é a relação de Diofanto de Alexandria.

Uma forma mais simples do que a relação de Diofanto de Alexandria encontramos em Caraça (1951, p.22) a figura seguinte para justificar que (a + $c)-b=a-(b-c)$ :

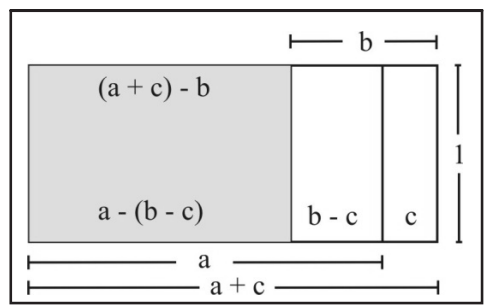

Figura 2 - modelo geométrico que mostra que $(a+c)-b=a-(b-c)$

Na Figura 2 observamos que a altura do retângulo mede 1 unidade de medida, a área do retângulo sombreado vale $(\mathrm{a}+\mathrm{c})-\mathrm{b}=\mathrm{a}-(\mathrm{b}-\mathrm{c})$ unidades de área.No interior da justificação desta propriedade associativa também está a justificação de que “ $-x-=+$ ", uma vez que, devemos ter $(a+c)-b=a-(b$ $-\mathrm{c})=\mathrm{a}-\mathrm{b}+\mathrm{c}$.

Em Pires, Curi e Pietropaolo (2002) a apresentação da regra dos sinais para a multiplicação, para os casos em que ambos os fatores são negativos, é 
feita por meio de uma apresentação geométrica de cálculo de área (ou cálculo do número de retângulos) inspirada na representação geométrica da relação de Diofanto para o caso particular $-2 \times-1=2$.

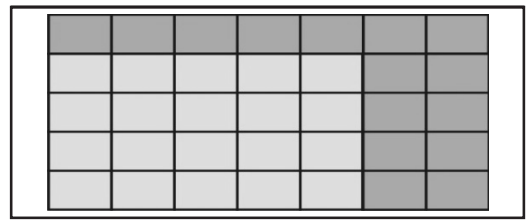

Figura 3 - modelo geométrico que explica que $-2 \times-1=2$

Os autores, a partir da Figura 3,observam que a área do retângulo da região mais clara é 20 e é dada por:

$$
\begin{gathered}
(7-2) \times(5-1)=20 \\
(+35)+(-7)+(-10)+(\Delta)=20 \\
(+18)+(\Delta)=20
\end{gathered}
$$

A partir desta situação, concluem que $\Delta=+2=-2 \times-1$.

\subsection{Modelos didáticos baseados na ideia de balanço: o modelo comercial}

O modelo comercial, denominado assim por Glaeser (1981), baseia-se em problemas de balanço do tipo crédito/débito, ganho/perda, sobe/desce, presente/futuro, enche/esvazia etc. São modelos concretos, bastante comuns, que associam, por exemplo, ao sinal “+” o lucro e ao sinal “-”" o prejuízo. Assim, alguém que perdeu $\mathrm{R} \$ 8,00$ em um dia e no dia seguinte perdeu $\mathrm{R} \$ 8,00$, perde ao todo $\mathrm{R} \$ 16,00$. As contas são feitas da seguinte maneira: $-8+(-8)=-16$ ou $2 \times(-8)=-16$.

Neste tipo de modelo, para explicar o caso, por exemplo, “ $-\times-=+$ ", pode utilizar a ideia de tempo, associando ao sinal "-"o passado e ao sinal "+" o tempo presente.

Encontramos vários modelos do tipo comercial, com pequenas variações na contextualização de um autor para outro. Um exemplo bem característico deste tipo é encontrado em Bongiovanni, Leite e Laureano (1996, p.38) da seguinte maneira:

Quanto vale (-3). (-2)?

Um comerciante contabiliza os seus lucros da seguinte 
maneira:

- um sinal positivo para as quantidades que representam um lucro e um sinal negativo para as que representam um prejuízo;

- um sinal positivo para representar o tempo futuro e sinal negativo para representar o tempo passado.

Se esse comerciante perde $\mathrm{R} \$ 3,00$ por dia, veja como ele calcula quantos reais vai ter perdido daqui a dois dias (futuro): $(-3) \cdot 2=-6$.

Daqui a dois dias vai ter perdido $\mathrm{R} \$ 6,00$.

Se esse comerciante perde $\mathrm{R} \$ 3,00$ por dia, veja como ele calcula quantos reais a mais tinha dois dias antes (passado): $(-3) \cdot(-2)=6$

Dois dias antes este comerciante estava $\mathrm{R} \$ 6,00$ mais rico.

Esses autores tomam por base este exemplo para concluir que o produto de dois números negativos é um número positivo.

Guelli (2001, p. 26) apresenta, para explicar o produto de inteiros, um modo que caminha no sentido da explicação dada anteriormente. É o seguinte: Imagine uma caixa-d'água, com capacidade de 720L, inicialmente vazia, sendo cheia com uma mangueira que despeja $20 \mathrm{~L}$ de água por minuto na caixa.

- Após 36 minutos, +36 , a caixa estará cheia: $(+20)(+36)=$ 720

- Há 18 minutos, -18, faltavam 360L para encher a caixa: $(+20)(-18)=+360$

Imagine esta mesma caixa-d’água, agora cheia, sendo utilizada para irrigar os campos. Seis litros por minuto estão saindo da caixa: -6 .

- após 36 minutos, +36 , a caixa terá $216 \mathrm{~L}$ a menos: $(-6)(+36)$ $=-216$.

- Há 18 minutos, -18, havia 108L a mais na caixa: $(-6)(-18)=$ +108 .

Estes autores concluem que: se dois números são diferentes de zero, e possuem o mesmo sinal, o produto deles é o produto de seus módulos: $\mathrm{ab}=$ $|\mathrm{a}| \cdot|\mathrm{b}|$.

O modelo apresentado é um modelo do tipo comercial, de ganho/perda, no caso aqui, o ganho e perda são substituídos pela dupla enche/esvazia, pela quantidade de água que enche ou sai de reservatório com o uso dos sinais negativo e positivo para indicar, respectivamente, o tempo passado e tempo presente. 


\subsection{Modelos didáticos que se baseiam no prolongamento da reta numérica dos naturais}

Se admitirmos que o zero é um número natural, então $\mathrm{N}=\{0,1,2,3, \ldots\}$ define o conjunto dos naturais. A reta numérica dos Naturais, que compreende o zero e os números positivos à direita de zero, são complementados por um conjunto de novos números à esquerda, os números inteiros negativos:

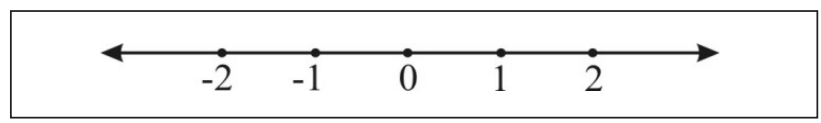

Figura 4 - reta numérica dos inteiros

Na reta numérica o crescimento se dá da esquerda para a direita. A manutenção desta ideia justifica, neste modelo, a regra do sinal, como por exemplo, na sequência seguinte:

$$
-1 \times 2=-\mathbf{2},-1 \times 1=-\mathbf{1},-1 \times 0=\mathbf{0},-1 \times-1=+\mathbf{1},-1 \times-2=+\mathbf{2}
$$

Em Jakubovic, Lellis, Centurión (1999, p. 40), no livro para a $6^{\text {a }}$ série (atualmente $7^{a}$ série), há a apresentação de uma tabela de multiplicação para explicar o prolongamento dos naturais aos inteiros da seguinte forma:

Observe esta tabela: os primeiros fatores decrescem 1 unidade, ao passo que os produtos crescem 3 unidades. Se isso continuar valendo, a tabela prosseguirá assim:

Tabela 1: a multiplicação de dois números negativos resulta em um positivo

\begin{tabular}{c|rrr|c} 
& 4. & $(-3)=$ & -12 & \\
Diminui & unidade & $(-3)=$ & -9 & \\
$\Downarrow$ & 2. & $(-3)=$ & -6 & \\
1. & $(-3)=$ & -3 & Aumenta \\
0. & $(-3)=$ & 0 & unidades \\
& $(-1)$. & $(-3)=$ & 3 & $\Downarrow$ \\
& $(-2)$. & $(-3)=$ & 6 & \\
$(-3)$. & $(-3)=$ & 9 &
\end{tabular}

Fonte: (JAKUBOVIC, LELLIS, CENTURIÓN, 1999, p. 40). 
Esses autores concluem dizendo que isso dá a ideia de que a multiplicação de dois números negativos resulta em um número positivo, e apresentam a seguinte regra prática para a multiplicação de dois números inteiros quaisquer:

- Multiplicamos os seus módulos.

- O produto será positivo se os dois fatores tiverem os sinais iguais e será negativo se os dois fatores tiverem sinais diferentes.

\subsection{Modelo do tipo lógico}

Este modelo, já comentado anteriormente, encontrado em Euler, se baseia em um argumento do tipo "lógico para explicar a regra do sinal " $-\times-=+$ " que é o caso mais emblemático. Para os demais casos e para a regra dos sinais para a adição, ele utiliza o modelo do balanço de ganho/perda. Para o caso " $-\times-=$ +", ele usa o seguinte argumento: uma vez que a regra da multiplicação para os casos " $+x+=+", "-x+=-"$ e" $+x-=$ " resulta em dois negativos e apenas um positivo para haver equilíbrio na quantidade dos resultados de sinais positivos e negativos, devemos ter então " $-\times-=+$ ".

Tal modelo não foi encontrado em nenhum dos manuais escolares consultados, mas é possível que faça parte do discurso do professor quando do desenvolvimento do assunto em aula: se tem um resultado positivo $(+\times+=$ + ) e dois negativos $(-\times+=-,+\times-=-)$ o outro $(-\times-)$ só pode dar positivo.

\section{0 ensino da regra dos sinais é possível no ensino fundamental?}

O Teorema de Hankel resolve o problema da regra dos sinais definitivamente do ponto de vista matemático, mas restam muitas questões didáticas a ele relacionadas. Citemos, por exemplo, o estudo de Glaeser (1981) que identifica diversas dificuldades relacionadas à compreensão dos números relativos: Inabilidade em manipular e dar sentido às quantidades negativas; prolongamento da reta numérica para incluir os negativos; a ambiguidade dos dois zeros (medida e origem); dificuldade de superar o sentido concreto, em oposição ao sentido formal, da noção de negativo; a busca de um modelo explicativo unificador.

Em 1867, Hankel dá a resposta matemática definitiva para a questão, com o seguinte resultado: a única multiplicação nos reais que prolonga a multiplicação usual sobre $\mathrm{R}+$, respeitando as distributividades à esquerda e à 
direita é aquela que obedece a regra usual dos sinais para a multiplicação ${ }^{4}$ :

\begin{tabular}{|c|}
\hline$+\times+=+$ \\
\hline$+\times-=-$ \\
\hline$-\times+=-$ \\
\hline$-\times-=+$ \\
\hline
\end{tabular}

Quadro 1 - Regra usual

Quer dizer, para manter tais propriedades com os reais negativos devemos definir a regra para os sinais da maneira usual, como fazemos na escola quando abordamos os números relativos.

\section{Análise didática, em temos de congruência semântica, dos modelos do tipo comercial, do modelo de área baseado na relação de Diofanto de Alexandria e do prolongamento da reta numérica dos naturais}

Coquin-Viennot (1985, p. 159) afirma que os manuais escolares franceses, de 1970 a 1975, apresentavam os números relativos a partir do modelo comercial. No Brasil, modelos baseados em balanço de duplas, como por exemplo, o tipo ganho/perda, possivelmente são os mais comuns. Apresentamos, neste texto, apenas dois daqueles que encontramos em vários manuais escolares, uma vez que nos interessamos, neste estudo, pela variedade e não pela quantidade de modelos.

\subsection{O modelo comercial}

A regra do sinal para a multiplicação é artificial, pura invenção da mente humana. O Teorema Hankel faz cair por terra a ideia da existência de um bom modelo de explicação. Por exemplo, o modelo comercial pode se tornar um obstáculo à compreensão das propriedades multiplicativas. Neste modelo, o aluno pode se convencer facilmente, na situação, $-3+4=+1$, associando -3 a uma dívida e +4 a um ganho, mas dificilmente será convencido do mesmo em $-4 \times-$ $2=+8$.

\footnotetext{
${ }^{4}$ Demonstração: $0=\mathrm{a} .0=\mathrm{a} \cdot(\mathrm{b}+\mathrm{op} \mathrm{b})=a \cdot b+a . o p b$. (op $\mathrm{x}$ indica o oposto aditivo de $\mathrm{x}$ ). $0=0 . o p \mathrm{~b}=($ op $\mathrm{a}+\mathrm{a}) . \mathrm{op} \mathrm{b}=$ op $a . o p b+a . o p b$. Obtemos, portanto, comparando estes dois resultados em itálico, que $\mathrm{ab}=$ op $\mathrm{a}$. op $\mathrm{b}$.
} 
O modelo comercial pode auxiliar o aluno a resolver problemas no campo aditivo, tais como aqueles que aparecem nos manuais escolares, mas pode se tornar um obstáculo à resolução de problemas no campo multiplicativo. No entanto, a análise didática deste modelo, em termos da noção de congruência semântica introduzida por Duval $(1988,1995,1999,2004)$ nos permite fazer um alerta mesmo para o campo aditivo:

Duas expressões podem ter o mesmo sinônimo ou referencialmente equivalentes (elas podem "dizer a mesma coisa", elas podem ser verdadeiras ou falsas conjuntamente) e não serem semanticamente congruentes: neste caso há um custo cognitivo importante para a compreensão (DUVAL, 1988, p. 8).

São três os critérios para indicar se duas expressões são semanticamente congruentes (DUVAL, 2004, p. 79 - 93):

CRITÉRIO 1: Correspondência entre as unidades significativas própria a cada registro; CRITÉRIO 2: Univocidade para cada unidade significativa a ser convertida. Para uma unidade de partida, não há mais de uma unidade (significativa) possível no registro de chegada. CRITÉRIO 3: a ordem na organização das unidades significaticas de partida é conservada na representação de chegada.

Para entender um pouco melhor o que este autor quer dizer na citação, comecemos com o exemplo a seguir:

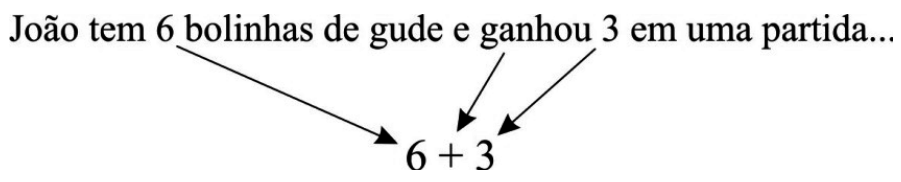

Figura 5 - Congruência semântica entre frase e expressão aritmética

Podemos constatar, conforme indicamos nestes registros na Figura 5, que frase e expressão algébrica são semanticamente congruentes, segundo os três critérios. As três unidades significativas na frase são: tem 6 bolinhas, ganhou e 3 que correspondem, na mesma ordem, com as três unidades significativas na expressão aritmética: "6”; “+” e "3”. A frase e a expressão aritmética possuem 
também equivalência referencial.

Já a frase Um pai (p) tem 33 anos a mais do seu filho ( $f$ ) possui congruência semântica, segundo os três critérios acima, com a expressão "p + 33 = f'. No entanto, frase e expressão não são referencialmente equivalentes.

Esta mesma frase não possui congruência semântica com a expressão " $\mathrm{p}-\mathrm{f}=33$ ", mas a frase e expressão aritmética são referencialmente equivalentes: esta última é uma expressão (outra poderia ser " $p=33+\mathrm{f}$ ”) que pode conduzir à resolução correta do problema. Tanto " $\mathrm{p}-\mathrm{f}=33$ " quanto " $\mathrm{p}=$ $33+\mathrm{f}$ " são referencialmente equivalentes, mas não são congruentes com a frase referida.

Problemas discursivos que são semanticamente congruentes com a expressão matemática, mas que não são referencialmente equivalentes, levam a uma taxa muita baixa de sucesso; da mesma forma acontece com problemas que são referencialmente equivalentes, mas não são semanticamente congruentes. A resolução de problemas que solicitam a passagem de um registro discursivo para um registro aritmético ou algébrico exige a equivalência referencial.

As associações à operação de adição ao verbo ganhar, adquirir, ter a mais, creditar etc. e à operação de subtração ao verbo perder, endividar, desaparecer, carregar, levar, gastar, debitar etc., poderão acarretar dificuldades na resolução de certos tipos de problemas ou em situações em que estas associações nem sempre se traduzem em equivalência referencial.

\subsection{Modelo de área baseado na relação de Diofanto de Alexandria}

No caso do modelo geométrico apresentado por Pires, Curi e Pietropaolo (2002), já citado anteriormente, baseado no cálculo de área (Figura 3) temos, tomando por base as três relações apresentadas

$$
\begin{aligned}
& \text { relação (a) } \\
& \text { relação (b) } \\
& \text { relação (c) }
\end{aligned}
$$

$$
\begin{gathered}
(7-2) \times(5-1)=20 \\
(+35)+(-7)+(-10)+(\Delta)=20 \\
(+18)+(\Delta)=20
\end{gathered}
$$

as seguintes observações:

- a relação (a) é baseada no balanço de área ou na contagem de retângulos da Figura 3 que apresenta congruência semântica e equivalência referencial com a figura; 
- a relação (b) é obtida pelos autores por aplicação da propriedade distributiva na relação (a).

O balanço de área neste ponto do desenvolvimento da ideia não é mais usado, uma vez que não mais mantém sentido, em termos de área ou quantidade de retângulos, com as quantidades $-7,-10$ e $\Delta$. Além disso, cabe um questionamento do porque dos sinais " + " antes de $(-7)$ e (-10): não equivalem ao uso da comutatividade dos sinais "+" e "-" na multiplicação?

O sinal "+" antes de " $(\Delta)$ " na relação (b) e mantido na relação (c) equivale ao uso da regra " $-x-=+$ " a qual os autores pretendem verificar?

Uma alternativa para esta relação é obter outra equivalente, ainda com o balanço de área na Figura 3. Assim, é possível escrever: $20=7 \times 5-7 \times 1-5 \times 2$ +2 .Um aspecto, aqui, é fundamental destacar: a aparição de “+ 2", que pode ser explicada por um processo simples de contagem dos retângulos na figura: na contagem do número de retângulos, 2 deles (destacados na parte superior à direita da Figura 5, a seguir) são acrescentados devido ao fato de que esses retângulos foram retirados duas vezes: uma vez em $1 \times 7=7$ (primeira linha) e novamente em $5 \times 2=10$ (duas últimas colunas):

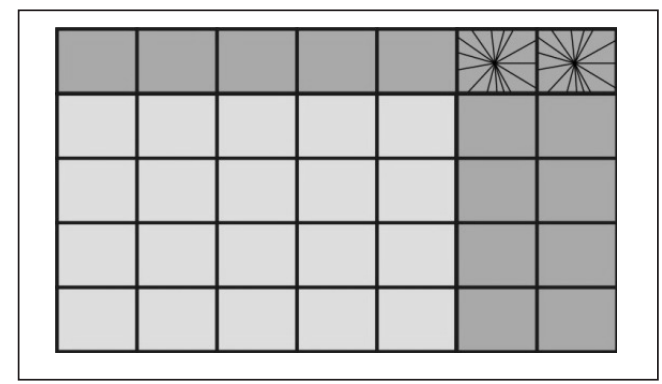

Figura 6 - destaque dos 2 retângulos que aparecem acrescentados em "35-7-10+2"

A Figura 6 tem um suporte importante, funciona como representação auxiliar, assim denominada por Duval (1999, p. 57-62) uma vez que permite tratamentos que podem ser confrontados com as operações na expressão (7 2) $\times(5-1)$ que é a representação principal. A representação auxiliar da figura tem um papel heurístico, daí a sua importância para a obtenção da relação

$$
(7-2) \times(5-1)=35-7-10+2
$$

apenas com a contagem de retângulos. 
A partir da relação (a), apenas com o uso da distributividade, podemos obter a relação seguinte

$$
(7-2) \times(5-1)=7 \times 5-7 \times 1-2 \times 5-2 \times-1=35-7-10-2 \times-1
$$

que é semanticamente congruente com o balanço de retângulos na figura. A comparação dessa última igualdade com a igualdade anterior permite escrever que " $-2 \times-1=+2$ ".

A contagem do número de retângulos em uma figura geométrica, que é bastante intuitiva e elementar, é confrontada por um desenvolvimento aritmético para constatar a aplicação da regra do sinal de multiplicação apenas para o caso “ $-2 \times-1=+2$ ". Neste procedimento, a constatação da regra dos sinais para o caso " $-\times-=+$ " se dá para um caso particular e a distributiva é utilizada em um único sentido.

\subsection{Modelo do prolongamento da reta numérica dos naturais}

A reta numérica (Figura 4) é uma representação auxiliar muito produtiva para os números relativos no campo aditivo. Esta reta possui congruência semântica com as operações de adição, como para os exemplos, bastante comuns, do tipo "-a + b", "-a + (-b)" e "a + (-b)".

A subtração, por exemplo, para o caso "a - (-b)" só poderá ser explorada no campo multiplicativo. Como explicar o uso da reta dos inteiros para a obtenção de 5 em " $2-(-3)=5$ ”? Se "-”" indica movimento na reta numérica à esquerda, como é que dois movimentos no sentido à esquerda vão resultar em um único movimento à direita? $\mathrm{O}$ desenvolvimento dessa expressão se dá da seguinte maneira: $\mathrm{a}-(-\mathrm{b})=\mathrm{a}+\mathrm{b}$. O caso " $\mathrm{a}-(+\mathrm{b})$ " também teria o desenvolvimento seguinte: $\mathrm{a}-(+\mathrm{b})=\mathrm{a}+(-\mathrm{b})$. O que observamos, nestas duas situações típicas, é o uso da regra dos sinais para a multiplicação.

A reta numérica dos inteiros é um suporte de representação, que pode ter um papel auxiliar importante na resolução de problemas aditivos (DUVAL, 1999, p. 57-62).

\section{Uma hierarquia na concepção dos relativos}

A partir de uma série de questões aplicadas (14 questões no total, algumas delas com vários subitens) a um grupo de 366 alunos (nível equivalente, no Brasil, às quatro últimas séries do ensino fundamental, com idade entre 13 e 16 
anos), Coquin-Viennot (1985, p. 176-181) estabelece a hierarquia seguinte nas concepções dos alunos a propósito dos relativos:

Nível I - concepção em que os relativos são tratados como naturais. $\mathrm{O}$ número é considerado como uma quantidade ou medida e deste modo só pode ser positivo. A noção de ordem não é completamente adquirida.

Neste nível, encontramos respostas do tipo " $2<5<6<7$ " e " $-2<5<$ $6<7$ ", que utilizam a ordem como se fossem naturais e, ainda, $-2<-7<5<6$ em que os negativos são classificados separadamente e na frente dos positivos, mesmo com $-2<-7$, para o exercício proposto de classificação numérica nos relativos seguintes: "Classificar em ordem crescente (utilizando o sinal $<$ ): $-7,5$, $-2,6 . "$

Nível II - nesta concepção para os problemas aditivos, os alunos utilizam os naturais, se possível, e se permitem obter uma resposta. A resolução de problemas multiplicativos nos relativos é apenas esboçada. Os negativos são tratados separadamente dos positivos. Portanto, ainda não há uma unificação do conjunto dos relativos.

Por exemplo, neste nível de concepção, encontramos " $\mathrm{x}-2<\mathrm{y}-3$ " como resposta ao problema aditivo de ordenação: "x e y são dois números tais que $\mathrm{x}>\mathrm{y}$. Escrever a relação de ordem para $\mathrm{x}-2$ e y -3 ”. Os alunos se prendem a presença de " 2 " e " 3 " $(2<3)$ para indicar a resposta. Para a resposta " $-3 \mathrm{x}>-3 \mathrm{y}$ " para o problema multiplicativo: "x e y são dois números tais que $x>y$. Escrever a relação de ordem para $-3 x$ e $-3 y$ ", os alunos se prendem ao fato que " $x>y$ " e que " -3 " aparece em ambos os lados da desigualdade. Observamos, nesses dois casos, que há congruência semântica entre resposta e texto do problema, mas não há equivalência referencial.

Para o exercício "Calcule $\mathrm{S}=2-2+0-1-3+5$ " os alunos deste nível somam separadamente os números positivos e negativos $(7-6)$ sem a simplificação dessas somas.

Nível III - neste nível de compreensão, a noção de ordem é estabelecida. Os problemas aditivos são resolvidos nos inteiros relativos. Pode-se considerar que a reta numérica é unificada, mas os problemas multiplicativos não são ainda completamente resolvidos.

Em relação aos três problemas anteriormente referidos, de ordenação numérica e de ordenação no campo aditivo são corretamente respondidos, mas não é o caso, ainda, para o problema multiplicativo de ordenação.

O Nivel IV corresponde a um grupo muito pequeno de alunos; o conjunto dos inteiros é utilizado para resolver mesmo os problemas que têm uma solução nos naturais. Os problemas multiplicativos são assimilados. 
Com base nesta hierarquia, esta autora conclui que:

(...) este modelo comercial tão prático e que é reforçado durante todo o começo da aprendizagem e que se instala definitivamente no espírito do aluno não mais como um modelo, mas como uma concepção dos relativos (níveis II e III). (COQUIN-VIENNOT, 1985, p. 184).

\section{Conclusões Finais}

Coquin-Viennot (1985) comenta que Michelot (1966) ${ }^{5}$ procurou longamente demonstrar que a noção de número relativo só pode ser atingida ao nível do pensamento formal e confessa:

Aderimos plenamente a esta noção dos relativos que só podem ser definidos ao nível formal. Nestas condições, não se está introduzindo um falso contrato didático quando se utiliza o modelo concreto para apresentar o conjunto dos números relativos? (COQUIN-VIENNOT, 1985, p. 183)

A conclusão desses autores é de que a noção dos relativos só pode ser atingida ao nível formal; as dificuldades assinaladas por Glaeser (1981), principalmente aquela relacionada à superação do sentido concreto em oposição ao sentido formal da noção de negativo, reforçam a ideia de que o ensino dos números negativos deve contar com o princípio de extensão em matemática em que o Teorema de Hankel é um exemplo desses princípios.

Diferentemente do que ocorreu com os naturais, em um processo de extensão que se desvinculou das coisas para se tornar algo puramente aritmético, encontramos, ainda, na concepção dos alunos, uma característica que os números negativos carregaram até o Século XIX:

[...] os matemáticos entendiam o número como coisa, como grandeza, como objeto dotado de substância. Tal concepção torna difícil a compreensão do número negativo ser menor que zero, uma vez que número é quantidade e o zero é a ausência de quantidade (PONTES, 2010, p. 19).

A regra dos sinais para a multiplicação coloca o professor frente a um tipo de dilema: ele está diante de uma regra estabelecida cuja explicação, o Teorema Hankel, citado anteriormente, ultrapassa a capacidade de compreensão

${ }_{5}^{5}$ Michelot, A. La notion de zéro. Paris : Vrin, 1966. 
do aluno do ensino fundamental. Além disso, as explicações alternativas, encontradas em manuais escolares, podem criar problemas como aqueles relacionados à congruência semântica, e que se mostram duradouros para o aluno. O que fazer? A seguir, reexaminaremos com mais cuidado o significado deste teorema e, a partir daí, tentaremos encontrar indicações de ensino dos relativos para o nível fundamental.

Consideremos a regra dos sinais usual para adição e a regra dos sinais para a multiplicação definida em cada uma das colunas da tabela a seguir, que será aplicada a um exemplo construído com o objetivo de explorar as distributividades à direita e à esquerda:

\begin{tabular}{|c|c|}
\multicolumn{1}{c}{ Regra usual } & Regra 2 \\
\hline$+x+=+$ & $+x+=+$ \\
\hline$+x-=-$ & $+x-=-$ \\
\hline$-x+=-$ & $-x+=-$ \\
\hline$-x-=+$ & $-x-=-$ \\
\hline
\end{tabular}

Quadro 2 - A regra usual e outra regra de sinais

Observemos que na Regra 2 colocamos que " $-x-=-$ " o que é diferente do que está definido na regra usual. Apliquemos estas duas regras à expressão $(1-3) \times(-5+1)$ :

\begin{tabular}{|c|c|c|}
\hline$(1-3) \times(-5+1)$ & Cálculo com a Regra usual & Cálculo com a Regra 2 \\
\hline Eliminando ambos os parênteses & $-2 \times-4$ & $-2 \times-4$ \\
& $=+8$ & -8 \\
\hline & $-2 \times(-5+1)$ & $-2 \times(-5+1)$ \\
Eliminando o parêntese à esquerda & $-2 \times-5-2 \times(+1)$ & $=-2 \times-5-2 \times(+1)$ \\
e usando a distributividade & $=10-2$ & $=-10-2$ \\
& $=+8$ & $=-12$ \\
\hline & $(1-3) \times-4$ & $(1-3) \times-4$ \\
Eliminando o parêntese à direita e & $1 \times-4-3 \times(-4)$ & $=1 \times-4-3 \times(-4)$ \\
usando a distributividade & $=-4+12$ & $=-4-12$ \\
& $=+8$ & $=-16$ \\
\hline
\end{tabular}

Quadro 3 - comparação entre duas regras de sinais para a multiplicação

Esta tabela mostra claramente que os resultados obtidos com a Regra usual são os mesmos, e isto acontece independente do modo de calcular: eliminando primeiro os parênteses ou aplicando a propriedade da distributiva à esquerda e a distributiva à direita. $\mathrm{O}$ mesmo não se pode dizer dos resultados obtidos com a Regra 2 que são diferentes entre si conforme o modo de calcular, 
e diferentes também daqueles calculados tendo por base a Regra usual.

Observamos que os cálculos com as expressões do tipo $(a-b) \times(b-c)$, como é o caso do exemplo tratado no Quadro 3, vão produzir os mesmos resultados conforme os três modos de calcular desta tabela com a Regra usual.Se fizermos a exigência de que $\mathrm{a}>\mathrm{b}$ e $\mathrm{c}>\mathrm{d}$, obteremos os mesmos resultados nos três modos de operar também com a Regra 2.Este fato é intrigante, porque são sob estas condições, conforme afirma Ball (1960, p. 106), que Diofanto de Alexandria expressa a relação $(a-b)(c-d)=a c-a d-b c+b d$.

Coquin-Viennot (1985), ao apresentar a sua classificação hierárquica de concepção pelo aluno de número negativo, enfatiza o quanto o modelo comercial impregna a concepção de números relativos. É um modelo forte, que pode apresentar sérios problemas até mesmo para o campo aditivo, problemas relacionados à congruência semântica, pode tornar-se um forte obstáculo à compreensão dos problemas multiplicativos.

Dentre as várias possibilidades de regra dos sinais para a multiplicação, a escolha recaiu sobre aquela regra que preserva as distributividades à esquerda e a direita. Estas propriedades já são observadas longamente na história, desde Diofanto de Alexandria ( $3^{\circ}$ séc. a. C.), quando tratadas com os números positivos e é bastante natural procurar prolongá-las também para o caso dos negativos. Esta ideia de prolongamento baseia-se em um princípio que, em parte, orienta o trabalho intelectual do homem, que Caraça (1951), já citado anteriormente, denomina de princípio de extensão. Acreditamos que seja este um dos aspectos, a ideia do princípio da extensão ausente do ensino fundamental e médio que deve guiar o ensino da regra dos sinais:

- para campo aditivo, modelo do prolongamento dos números naturais para a reta numérica dos inteiros como sugerido em (BRASIL, 1998b) que não deixa de ser uma aplicação do princípio de extensão. A reta numérica dos relativos é um suporte importante na resolução de problemas neste campo;

- para o campo multiplicativo, o modelo baseado no Teorema de Hankel, que tem por base a ideia do princípio da extensão da propriedade da distributividade dos números positivos para o caso dos números negativos. A subtração será trabalhada neste campo para que as relações do tipo "a - (-b)" e "a $-(+b)$ " possam ser tratadas.

O que percebemos no exemplo apresentado no Quadro 3 é que se quisermos manter o mesmo resultado, independente do modo de calcular, devemos eliminar a possibilidade de que a regra para a multiplicação seja definida, por exemplo, conforme a Regra 2. Procedendo com a escolha de exemplos judiciosos 
podemos estabelecer a regra dos sinais para a multiplicação, examinando todos os casos possíveis de sinais: como o caso " $+x+=+$ " já é estabelecido com os números positivos e, deste modo, dos dezesseis casos possíveis restam apenas oito para serem testados. É interessante observar que, mesmo tratando-se de exemplos particulares, o procedimento que explora todas as possibilidades de regra dos sinais faz com que sejam eliminadas as situações em que as igualdades não são verificadas nos três modos de calcular como apresentados no Quadro 3: por esta razão, a Regra 2 dos sinais é descartada, e qualquer outra regra, até ficarmos com alguma (temos que optar por uma delas), no caso a Regra usual, como sendo a única que se mantém.

\section{Referências}

BALL, W.W. R. A short account of the history of mahematics. New York: Dover, 1960.

BRASIL. Parâmetros Curriculares Nacionais: Matemática ( $1^{\mathrm{a}}$ a $4^{\mathrm{a}}$ séries). Secretaria de Educação Fundamental. Brasília: MEC/CEF, 1998a.

BRASIL. Parâmetros Curriculares Nacionais: Matemática ( $5^{\mathrm{a}}$ a $8^{\mathrm{a}}$ séries). Secretaria de Educação Fundamental. Brasília: MEC/CEF, 1998b.

BONGIOVANNI, V.; LEITE, O. R. V.; LAUREANO, J. L. T. Matemática \& Vida. 6 a Série. São Paulo: Editora Ática, 1996.

CARAÇA, B. J. Conceitos fundamentais da matemática. Lisboa: Bertrand, 1951.

COQUIN-VIENNOT, D. Complexité mathématique et ordre d'aquisition: une hierarchie de conceptions à propos des relatifs. RDM, Grenoble, v.6, n. 2 - 3, p. 132 - 192, 1985.

COSTA, M. A. As idéias fundamentais da matemática e outros ensaios. São Paulo: Grijalbo e Editora da USP, 1971.

DIOFANTO DE ALEXANDRIA. La aritmética y el libro sobre los números poligonales. Tomo 1. Tradução de M. B. Muñoz; E. F. Moral; M. S. Benito. Tres Canto (Madrid): Nivola Libros Ediciones, 2007a.

DIOFANTO DE ALEXANDRIA. La aritmética y el libro sobre los números poligonales. Tomo II. Tradução de M. B. Muñoz; E. F. Moral; M. S. Benito. Tres Canto (Madrid): Nivola Libros Ediciones, 2007b. 
EULER, L. Vollständige Anleitung zur Algebra Opera Omnia. Series Prima. Volumen Primum, 1770.

DUVAL, R. Écarts semantiques et cohérence mathématique: introduction aux problems de congruence. Annales de didactique et de sciences cognitives,v. 1, IREM de Estrasburgo, 1988.

DUVAL, R. Sémiosis et pensée humaine: Registres sémiotiques et apprentissages intellectuels. Bern: Peter Lang, 1995.

DUVAL, R. Conversion et articulation des représentations analogiques. IUFM Nord Pas de Calais: Séminaires de Recherches, 1999.

DUVAL, R. Los problemas fundamentales en el aprendizaje de las matemáticas y las formas superiores en el desarrollo cognitivo. Tradução de Myrian V. Restrepo. C. Universidade del Valle. Valle: Merlin I. D., 2004.

GLAESER, G. Epistemologie des nombres relatifs. RDM, Grenoble, v. 2 n. 3, p. 303 $546,1981$.

GUELLI, O. Matemática: uma aventura do pensamento. São Paulo: Ática, 2001.

HOGBEN, L. Maravilhas da matemática: Influência e função da matemática nos conhecimentos humanos. 4. Ed. Tradução de P. M. da Silva. Rio de Janeiro: Editora Globo, 1956. v. 1.

JAKUBOVIC, J.; LELLIS, M.; CENTURIÓN, M. Matemática na medida certa. São Paulo: Editora Scipione, 1999.

KARLSON, P. A magia dos números: Matemática ao alcance de todos. Rio de Janeiro: Editora Globo, 1961.

MICHELOT, A. La notion de zéro. Paris: Vrin, 1966.

NATIONAL COUNCIL OF TEACHERS OF MATHEMATICS - NCTM. Princípios e Normas para a Matemática Escolar. Tradução de Magda Melo. Lisboa: Associação Portuguesa de Matemática, 2008.

PIRES, C. C.; CURI, E.; PIETROPAOLO, R. Educação Matemática. $6^{\text {a }}$ série. São Paulo: Atual, 2002.

PONTES, M. de O. Obstáculos superados pelos matemáticos no passado e vivenciados pelos alunos na atualidade: a polêmica multiplicação de números inteiros. 2010, $157 f$. 
Tese (Doutorado em Educação) - Universidade Federal do Rio Grande do Norte/ UFRN, Natal, 2010.

Submetido em Junho de 2011. Aprovado em Setembro de 2011. 